\title{
Design process of the CMS Silicon Tracker for Super-LHC
}

\author{
Carlo CIVININI*† \\ INFN - Firenze \\ E-mail: carlo.civinini@fi.infn.it
}

Following an expected increase of the LHC luminosity beyond its design value of $10^{34} \mathrm{~cm}^{-2} \mathrm{~s}^{-1}$ the CMS collaboration is planning for a series of upgrades on the experimental apparatus. In particular the silicon tracker should undergo two major upgrades to cope with the increased luminosity of the collider. The first intervention (Phase I) concerns a replacement of the pixel system, the rest of the tracker being untouched. A second major upgrade (Phase II) consists of a full replacement of the entire silicon tracker to cope with the foreseen ultimate SLHC luminosity $\left(10^{35}\right.$ $\mathrm{cm}^{-2} \mathrm{~s}^{-1}$ ). In these extreme conditions several hundred of interactions per beam crossing are expected and the number of charged particles generated will correspondingly increase with respect to the LHC conditions, requiring a new detector with a much higher granularity. This should be achieved keeping both the tracker power consumption and the material budget at levels which will not jeopardize the instrument operability and perfomance. Furthermore, the radiation tolerance of the new silicon sensors should be much higher than the present ones, requiring major developments on the detector technology side. Finally, the new tracker should be able to provide data to contribute to the CMS first level trigger. Possible layouts of the new tracking system have to be simulated in detail to understand the behaviour of the apparatus in a very high pile-up condition and to define a new optimal design which could meet the SLHC requirements.

In these proceedings the CMS silicon tracker upgrades will be described, with a special emphasis on Phase I pixel system, Phase II tracker and first level track trigger.

European Physical Society Europhysics Conference on High Energy Physics

July 16-22, 2009

Kraków, Poland

\footnotetext{
* Speaker.

On behalf of the CMS Collaboration.
} 


\section{Introduction}

The plan for the LHC luminosity upgrade [1] is divided into two parts: during 'Phase I' the collider will reach a peak luminosity within the range of $2-3 \times 10^{34} \mathrm{~cm}^{-2} \mathrm{~s}^{-1}$, then ('Phase II') an ultimate instantaneous luminosity of $10^{35} \mathrm{~cm}^{-2} \mathrm{~s}^{-1}$ is foreseen to be achieved.

Phase I luminosity will be gradually reached changing some of the CERN injector components and modifying the LHC interaction regions. The old CERN 'Linac 2' will be replaced by the new 'Linac 4' which will allow a higher injection energy into the 'Booster' and a better beam brightness. Furthermore new triplet quadrupoles, based on $\mathrm{Nb}$-Ti superconduction technology, will replace the present final focusing optics; the LHC collimators and separation elements will be also improved. The time schedule for this intermediate upgrade scenario depends on the LHC startup time; an educated guess could lead to a Phase I upgrade completion not before year 2014.

A step further in the luminosity upgrade will involve major changes in the LHC injector chain. Linac 4 will be upgraded to a higher energy machine called 'Low Power Superconducting Proton Linac', which will directly inject high intensity proton beams into a new $50 \mathrm{GeV}$ syncrotron replacing the old CERN PS. The Phase II scenario is still in an R\&D and design-optimization stage and will possibly be approved in the coming years.

The present silicon tracker system has been installed in CMS at the end of 2007; it has been commissioned and large samples of cosmic ray data for debugging and allignment have been acquired. Its structure and characteristics are fully described in [2].

\section{Phase I upgrade}

The present pixel detector [2] has been designed to withstand a fluence of $6 \times 10^{14} n_{e q} \mathrm{~cm}^{-2}$ : for the innermost detector layer this corresponds to a lifetime of two years at the full LHC design luminosity. The readout architecture of the present pixel system functions efficiently up to the highest rates expected when the LHC operates at full luminosity, but a factor 2-3 increase in luminosity would lead to an unacceptable deadtime in the readout chip. For these motivations and taking into account the LHC luminosity evolution, it is expected that the pixel system should be replaced in coincidence with the LHC Phase I upgrade.

The new CMS pixel system layout [3] will include a fourth barrel layer as well as a third endcap disk per side. The main features of this detector will be an aggressive reduction of the material budget (a factor three in weigth with respect to the present system) and a modificaton of the readout chip to allow for a full readout efficiency also at Phase I luminosities.

The innermost barrel layer radius will be reduced from $4.4 \mathrm{~cm}$ to $3.9 \mathrm{~cm}$, while the new fourth layer will be inserted at $16 \mathrm{~cm}$ to reduce the present radial gap between the last measured pixel point to the first strip layer improving the pattern recognition efficiency. The exact position of the three endcap disks are being optimized to assure a hermetic angular acceptance for $|\eta|<2$.4. This layout will allow to measure at least a triplet of three-dimensional high precision points at the beginning of a trajectory. This will help to reconstruct tracks with high efficiency keeping the fake rate at low level. The material reduction and the radial position of the innermost layer will allow for a better resolution of the transverse and longitudinal impact parameter measurement. The present $C_{6} F_{14}$ liquid cooling will be replaced by an evaporative $\mathrm{CO}_{2}$ system with ultra-light mechanics substan- 
tially reducing the material budget. The pixel readout chip will be slightly modified increasing the readout buffer depth to eliminate the dead time when the detector will operate at high luminosity.

\section{Phase II upgrade}

The luminosity increase expected during Phase II will require the replacement of the full CMS tracking system. To benefit from the expected increase of the collected integrated luminosity, the CMS tracker should maintain tracking and vertexing performance very similar to the one of the present detector. To achieve this goal the detector granularity must necessarily increase to allow for a robust track recognition. Higher granularity is also requested by the expected increase of the sensors leakage current after heavy irradiation. On the other hand an uncontrolled increase of the number of electronics channels would lead to a tracker dominated by power cables and cooling services with performance spoiled by the material budget. The usage of deep submicron CMOS technology for front-end electronics will guarantee radiation tolerance and a reduced power consumption. On the other hand the lower voltage required by this technology will imply higher currents and consequently heavier cables. This issue is the subject of intensive R\&D activities on novel powering schemes (rad-hard DC/DC converters or serial powering) aimed at a cost and material budget reduction of the electronics related items.

Last but not least the silicon sensors which can survive the very high radiation levels of the new collider are being investigated in various $R \& D$ packages both within the collaboration and in more general projects like RD50 [4]. The sensor technology to be used will be chosen taking into account not only the radiation hardness but also other system aspects like operational temperature or large scale availability.

\section{1 level-1 track trigger}

At the SLHC luminosities the CMS level-1 trigger rate will greatly exceed its maximum allowed badwidth of $100 \mathrm{kHz}$. In fact the single electron, muon and jet triggers will have rates which cannot be easily reduced increasing the transverse momentum $\left(p_{T}\right)$ thresholds and isolation criteria. Simulations done on the CMS High Level Trigger system (HLT) have demonstrated $[5,6]$ that adding tracking information on muon trajectories will reduce combinatorial background and will recover discrimination power on $p_{T}$. This argument can lead to the conclusion that including at level-1 one or more tracker points in a limited $r-\phi$ window could improve the muon $p_{T}$ resolution and reduce the fake triggers.

Moving all tracker data outside the detector for decision logic within the level-1 latency time is not feasible, so an 'on-detector' data reduction, to select hits generated by high momentum particles, is essential. Currently two different methods to obtain a track momentum estimate at the module level are under study. One [7, 8, 9] uses a single sensor and it is based on the measurement of the cluster width, the other $[10,11]$ uses modules built with two sensors very close to each other and electronics which correlates the particle hits on them.

The first method uses the charge distribution deposited into a sensor by the incident particle. If the particle momentum is low the trajectory at the detector has been considerably bent by the magnetic field and it crosses the sensor not perpendicularly to its surface (in the r- $\phi$ plane). In that way the charge is produced inside the sensor over an inclined path inducing signals on more than one strip 
or pixel. The other way around a high momentum particle is not bent significantly and its trajectory crosses the module at an angle very close to the perpendicular producing signals in a very limited number of strips or pixels. The second method uses two closely spaced sensors (order of millimeters) and makes use of the hit correlations in the two sensors to estimate the local trajectory angle. Here a set of segments is locally reconstructed in each module: back-extrapolating segments directions, a high momentum track is selected when it points to the interaction region.

\subsection{Tracker layouts}

The layout definition for Phase II is under way. Two different approaches have been selected: a design similar to the present tracker in the outer part containing specialized trigger stations and a 'full barrel' design where the entire tracker is realized by pixellated barrel-type trigger layers. Both systems foresee an inner pixel detector with a design very similar to the one under development for Phase I, but which will make use of a sensor technology able to survive at radii as low as $4 \mathrm{~cm}$.

The two layout geometries have now been implemented into the CMS simulation and reconstruction framework and are starting to produce results on comparisons between different tracking system strategies and designs. The reliability of the simulations depends on the accuracy of the detector model implemented: material budget for the tracker in general and for the new triggering stations in particular is crucial.

\section{Conclusions}

The LHC luminosity upgrade is presently divided into two parts. The CMS tracker collaboration follows the LHC luminosity evolution scheme for the detector upgrade plans. Phase I activities are currently well defined: a new pixel system with less material and more detection layers will replace the present one. For Phase II CMS is proceeding to develop a new tracker design using simulations to define the new layout. Important issues to be solved are a substantial reduction in the detector material budget and the possibility to include tracker data at the level-1 trigger.

\section{Acknowledgments}

I would like to thank all the colleagues involved in the CMS tracker construction, commissioning and upgrade activities and in particular those who helped me to prepare this contribution.

\section{References}

[1] E. Shaposhnikova, Intensity upgrade plans for CERN-LHC injectors, CERN-AB-2008-065 (2008).

[2] R. Adolphi et al., The CMS experiment at the CERN LHC, JINST. 0803, S08004, (2008).

[3] W. Erdmann et al., Upgrade Plans for the CMS Pixel Barrel Detector, submitted to Nucl. Instr. and Meth. A

[4] G. Casse, Overview of the recent activities of the RD50 collaboration on radiation hardening of semiconductor detectors for the sLHC, Nucl. Instr. and Meths. A (2009) 598, 54-60 
[5] The CMS Collaboration, CMS Data Acquisition and High-Level Trigger Design Report (2002) CERN/LHCC 02-26

[6] The CMS Collaboration, CMS Technical Design Report, Volume I: Detector Performance and Software, (2006) CERN/LHCC 2006-001

[7] G. Barbagli, F. Palla, G. Parrini, Track momentum discrimination using cluster width in silicon strip sensors for SLHC, In Prague 2007, Topical Workshop On Electronics For Particle Physics (TWEPP 07)

[8] F. Palla, G. Parrini, Tracking in the trigger: From the CDF experience to CMS upgrade, PoS VERTEX2007 034 (2007)

[9] F. Palla, Proposal for a First Level Trigger using pixel detector for CMS at Super-LHC, JINST 2 P02002 (2007) [doi: 10.1088/1748-0221/2/02/P02002]

[10] C. Foudas, A. Rose, J. Jones, G. Hall, A Study of a Tracking Trigger at First Level for CMS at SLHC, Proc. LECC 2005 Workshop, CERN Report CERN-2005-011(2005) 448-452

[11] J. Jones, A. Rose et al., Stacked Tracking for CMS at Super-LHC, Proc. LECC 2006 Workshop (2007) CERN-2007-001, 130-134 